\title{
BMJ Open Longitudinal associations between marine omega-3 supplement users and coronary heart disease in a UK population-based cohort
}

\author{
Marleen A H Lentjes, ${ }^{1}$ Ruth H Keogh, ${ }^{2}$ Ailsa A Welch, ${ }^{3}$ Angela A Mulligan, ${ }^{1}$ \\ Robert N Luben, ${ }^{1}$ Nicholas J Wareham, ${ }^{4}$ Kay-Tee Khaw ${ }^{5}$
}

To cite: Lentjes MAH, Keogh RH, Welch AA, et al. Longitudinal associations between marine omega- 3 supplement users and coronary heart disease in a UK populationbased cohort. BMJ Open 2017;7:e017471. doi:10.1136/ bmjopen-2017-017471

- Prepublication history for this paper is available online. To view these files, please visit the journal online (http://dx.doi. org/10.1136/bmjopen-2017017471).

Received 24 April 2017 Revised 21 June 2017 Accepted 31 July 2017

\section{CrossMark}

${ }^{1}$ Department of Public Health and Primary Care, University of Cambridge, UK

${ }^{2}$ Department of Medical Statistics, London School of Hygiene and Tropical Medicine, London, UK

${ }^{3}$ Department of Population Health and Primary Care, University of East Anglia, Norwich, UK

${ }^{4}$ MRC Epidemiology Unit, University of Cambridge, Institute of Metabolic Science, Cambridge, UK

${ }^{5}$ School of Clinical Medicine, University of Cambridge, Addenbrooke's Hospital, Cambridge, UK

\section{Correspondence to} Dr Marleen A H Lentjes; marleen.lentjes@phpc.cam. ac.uk

\section{ABSTRACT}

Objectives Assess the association between marine omega-3 polyunsaturated fatty acid ( $n-3$ PUFA) intake from supplements, mainly cod liver oil, and coronary heart disease (CHD) mortality.

Design Prospective cohort study, with three exposure measurements over 22 years.

Setting Norfolk-based European Prospective Investigation into Cancer (EPIC-Norfolk, UK).

Participants 22035 men and women from the general population, 39-79 years at recruitment.

Exposure Supplement use was assessed in three questionnaires (1993-1998; 2002-2004; 2004-2011). Participants were grouped into non-supplement users (NSU), n-3 PUFA supplement users (SU+n3) and non-n-3 PUFA supplement users (SU-n3). Cox regression adjusted for time-point specific variables: age, smoking, prevalent illnesses, body mass index, alcohol consumption, physical activity and season and baseline assessments of sex, social class, education and dietary intake (7-day diet diary).

Primary and secondary outcome measures During a median of 19-year follow-up, 1562 CHD deaths were registered for 22035 included participants.

Results Baseline supplement use was not associated with CHD mortality, but baseline food and supplement intake of n-3 PUFA was inversely associated with CHD mortality after adjustment for fish consumption. Using time-varying covariate analysis, significant associations were observed for SU+n3 (HR: $0.74,95 \% \mathrm{Cl} 0.66$ to 0.84 ), but not for SU-n3 versus NSU. In further analyses, the association for SU+n3 persisted in those who did not take other supplements (HR: $0.83,95 \% \mathrm{Cl} 0.71$ to 0.97 ). Those who became SU+n3 over time or were consistent $\mathrm{SU}+\mathrm{n} 3$ versus consistent NSU had a lower hazard of CHD mortality; no association with CHD was observed in those who stopped using n-3 PUFA-containing supplements.

Conclusions Recent use of n-3 PUFA supplements was associated with a lower hazard of CHD mortality in this general population with low fish consumption. Residual confounding cannot be excluded, but the findings observed may be explained by postulated biological mechanisms and the results were specific to $\mathrm{SU}+\mathrm{n} 3$.

\section{Strengths and limitations of this study}

- The use of dietary supplements was measured three times over the course of 22 years.

- The repeated measures enabled to study change in supplement use in relation to hard endpoints.

- The cohort was population-based as opposed to trials on omega-3 supplement use which have been in high-risk populations.

- Total omega-3 intake from both food (mainly fish sources) and supplement sources could be quantified; however, only for the baseline measure.

- Estimates were adjusted for sociodemographic and other behavioural risk factors, although residual confounding cannot be excluded.

\section{INTRODUCTION}

The traditionally high omega- 3 polyunsaturated fatty acid (n-3 PUFA) intake among the Inuit has been associated with a lower risk of ischaemic heart disease mortality in this population. ${ }^{1}$ Research leading on from this observed that in a Dutch cohort, where average fish consumption was $20 \mathrm{~g}$ /day, higher fish consumption was associated with lower relative risk of coronary heart disease (CHD) mortality between $1960-1980 .{ }^{2}$ A recent review of prospective cohort studies has observed a $16 \%(95 \%$ CI $5 \%$ to $25 \%)$ lower risk of CHD mortality in those consuming one portion of fish per week compared with lower frequency of consumption; however, null findings among included studies were equally observed. ${ }^{3}$ The association between fish intake and CHD has been attributed to n-3 PUFA, which are mainly derived from oily fish. According to the National Diet and Nutrition Survey in 2000/2001, less than $50 \%$ of the UK population consumed oily fish once a week ${ }^{4}$; however, dietary supplements may provide $50 \%$ of the n-3 PUFA intake. ${ }^{5}$ 
N-3 PUFA at pharmaceutical doses reduce plasma triglycerides and possibly thrombosis; whereas lower doses (around $250 \mathrm{mg} /$ day, obtainable from fish consumption and low dose supplements such as cod liver oil (CLO)) have been observed to lower the risk of sudden death or CHD death in trials and observational studies, which may be due to the influence of n-3 PUFA on heart rate and arrhythmia. ${ }^{6-8}$ Reviews of trials on n-3 PUFA supplementation in relation to overall cardiovascular diseases (CVD) ${ }^{9}$ or when separated into subgroups of stroke and coronary diseases, ${ }^{10-12}$ have shown no overall benefit, particularly in the most recent trials. It has been suggested that background medication, inclusion of non-fatal events as outcome measures and increasing trends in n-3 PUFA/ fish consumption might underlie the null findings. ${ }^{13} 14$

Although there have been a number of studies on the association between n-3 PUFA intake and CHD, the evidence for a protective association between n-3 PUFA supplement intake and CHD remains inconclusive. ${ }^{15}$ Consumption of n-3 PUFA supplements is traditionally high in Northern European countries. ${ }^{16}$ A Swedish cohort observed no association for fish oil supplements and CVD mortality ${ }^{17}$; whereas a study based in Iceland observed a lower risk of CHD hospitalisation with high frequency of CLO consumption. ${ }^{18}$ Previous studies have relied on single baseline measures of supplement use and covariates, ${ }^{19}$ excluded fish oil supplement users because of minimal use in the studied cohort ${ }^{20}$ or lacked detailed data on supplement use and therefore imputed n-3 PUFA content $^{21}$; moreover, trials have included participants at increased risk of $\mathrm{CHD}^{22-24}$ or included those who have already experienced a CHD event ${ }^{25-28}$; the effects of n-3 PUFA supplementation in a general population are less well documented, and the first primary prevention trial is ongoing. ${ }^{29}$

The aim of this analysis was to use three repeated measures of supplement use to investigate the association between n-3 PUFA supplement intake and CHD mortality in a general population-based cohort.

\section{METHODS}

An extended version of the methods has been included in online supplementary appendix I.

\section{Study design}

Recruitment for the Norfolk-based European Prospective Investigation into Cancer and Nutrition (EPIC-Norfolk) started in 1993 and was completed in $1998 .^{30} 31$ Thirty-five general practices in the Norfolk area of East Anglia (UK) took part in the study; 77630 registered patients were approached, 30445 consented. Exposures have been reassessed over the course of the study; participants completed general Health and Lifestyle Questionnaires (HLQ) and attended three health examinations (HE) up to 2011 (figure 1, online supplementary appendix I). Data on health outcomes and mortality were collected passively. Ethical approval for the study was given by the
Norwich District Health Authority Ethics Committee. Participants gave written informed consent.

\section{Assessment of $\mathrm{n}-3$ PUFA supplement use}

Dietary supplements were defined according to the EU directive 2002/46/EC. ${ }^{32}$ Prescribed medication containing minerals and/or vitamins were not considered supplements. The sum of eicosapentaenoic acid (EPA) and docosahexaenoic acid (DHA) from supplements were the main exposure, referred to as n-3 PUFA. We used data from three dietary supplement assessments (DSA), named DSA1, DSA2, DSA3. All covered a 1 week recall (online supplementary appendix I).

At each DSA, participants were grouped by the type of supplement they consumed:

- non-supplement users (NSU);

- non-n-3 PUFA supplement users (SU-n3): participants who consumed one or more supplements that did not contain n-3 PUFA;

- n-3 PUFA supplement users (SU+n3): participants who used n-3 PUFA supplements, either singly or in combination with other non-n-3 PUFA supplements.

Participants were also grouped into five categories identifying change in n-3 PUFA supplement use between two consecutive DSA (online supplementary appendix I and appendix II): 'consistent NSU', 'was SU+n3', 'became SU+n3', 'consistent SU+n3', 'other SU'.

Average daily nutrient composition from supplements was calculated using the Vitamin and Mineral Supplement system ${ }^{33}$ and added to the average daily food intake to obtain average total nutrient intake. Validation of these data with biomarkers were undertaken. ${ }^{5}$

\section{Dietary covariates}

The analyses adjust for potential dietary confounders measured at DSA1. Diet was assessed using a 7-day diet diary (7dDD) between 1993-1998. ${ }^{34}$ Data were entered by trained staff into DINER ${ }^{35}$ and checked and calculated by nutritionists using DINERMO. ${ }^{36}$ The underlying food composition tables were derived from McCance \& Widdowson's Composition of Foods and supplements. ${ }^{37}$ Missing values for fatty acids were further completed using a recipe calculation system. ${ }^{38}{ }^{39}$ The baseline $7 \mathrm{dDD}$ provided data on average daily energy intake (MJ/day), proportion of energy provided by macronutrients (\% en), the sum of EPA and DHA intake (g/day) referred to as 'n-3 PUFA' and consumption of the disaggregated amounts of fruit, vegetable, red meat, processed meat, white meat, and white and oily fish consumption (g/day) ${ }^{36}$

\section{Assessment of other covariates}

Social class was measured through occupational status (professional, managerial, skilled non-manual, skilled manual, semi-skilled, non-skilled). Highest education level achieved was divided in four categories (no qualification, O-level, A-level, degree). Both variables were derived from HLQ1 (coinciding with DSA1) and are timefixed. The remaining covariates are time-dependent and 


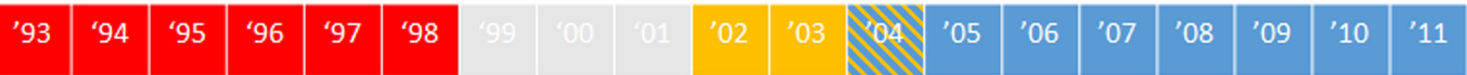

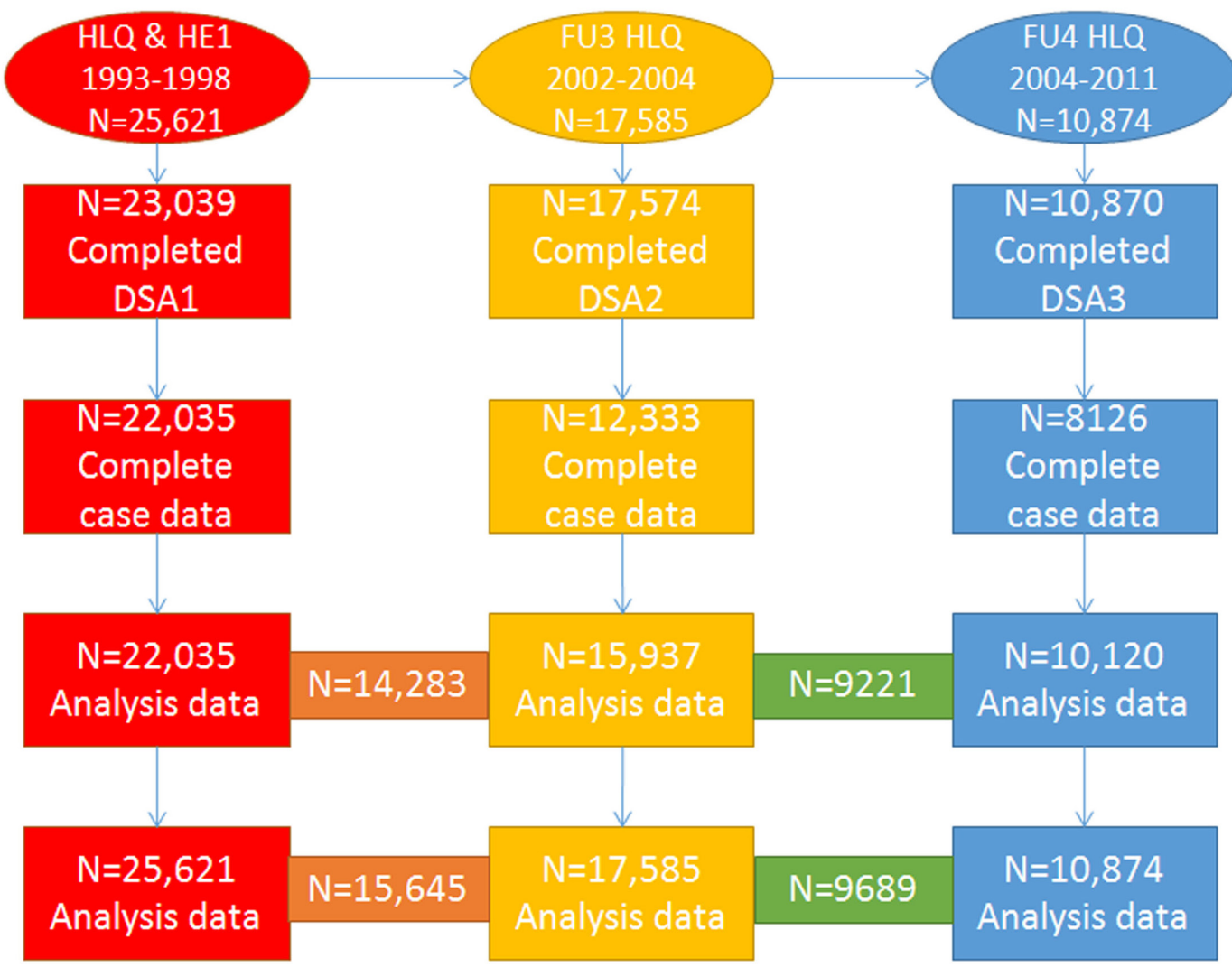

Loss due to missing supplement data

Loss due to missing covariable* data

Carry over data from previous time point if available

Using multiple imputation

Figure 1 Number of participants included at each DSA time point studied, with different scenarios of exclusion due to missing data. *Social class, education, smoking status, physical activity, body mass index, marital status and alcohol consumption. The numbers in the orange and green boxes relate to the number of participants available when the two consecutive DSA were considered in the analysis. The grey area in the timeline represents unobserved time. For more information regarding data availability see supplementary appendix I. DSA, dietary supplement assessment; FU, follow-up; HE, health examination; HLQ, Health and Lifestyle Questionnaire.

refer to measures obtained at time points corresponding to the DSA (online supplementary appendix I). We identified participants at higher risk of CHD using responses to the questions, "Has the doctor ever told you that you have [myocardial infarction/diabetes/stroke]?”. Participants were classified as a never smoker, former smoker or current smoker. Alcohol consumption was classified as none, $>0-14,>14-28,>28$ units/week. Marital status was recategorised into married (married/living as married) or non-married (widowed, divorced, separated, single). Participants were classified as being active, moderately active, moderately inactive or inactive. Body mass index (BMI) $\left(\mathrm{kg} / \mathrm{m}^{2}\right)$ was calculated from height and weight measured by a trained nurse.

\section{Participant selection, case ascertainment and outcomes studied}

Participants were eligible for analyses if they provided data on supplement use at any of the three time points (DSA1, DSA2, DSA3) and attended the corresponding HE (figure 1); 4030 participants were not included at any time point studied. The participant's National Health Service number (a unique national patient identifier) was linked to the data from the Office of National Statistics to obtain vital status and causes of death. A similar procedure was followed for causes of hospital admissions registered by the East Norfolk Primary Healthcare Trust which records for its residents the admissions in England and Wales. The main endpoint studied was CHD mortality mentioned anywhere on the death certificate, identified with International Classification of Diseases (ICD) $9410-414$ or ICD10 I20-I25. In sensitivity analyses, we investigated the use of different definitions for the CHD endpoint: (1) CHD mortality mentioned as underlying cause of death, (2) death due to acute myocardial infarction (ICD-codes 410 or I21) or (3) first recorded hospitalisation due to CHD.

\section{Statistical analysis}

A description of the cohort at DSA1, DSA2 and DSA3 is given. To study potential changes in supplement user characteristics over time, a multinomial logistic regression 
analysis was performed at each DSA with supplement use group as the outcome variables and the variables noted above as explanatory variables.

The main analyses used Cox regression, using the follow-up time scale. Individuals who died from causes other than the event of interest were censored at their date of death and individuals who did not die from any cause were censored at the end of follow-up (31 March 2015).

First, we modelled associations between supplement use at DSA1 and CHD mortality, with adjustment for covariates measured at DSA1. A series of cumulative adjustment models were used: sex and age-adjusted estimates (model 1); including smoking, BMI $\left(\mathrm{kg} / \mathrm{m}^{2}\right)$, physical activity, alcohol intake, social class, education and season in which the questionnaire was completed (model 2); including self-reported myocardial infarction, stroke or diabetes (model 3); including energy intake (MJ/day) and disaggregated fruit $(\mathrm{g} /$ day), vegetable $(\mathrm{g} /$ day), red meat and processed meat (g/day), and white meat (g/ day) (model 4); including white fish (g/day) and oily fish (g/day) (model 5). Second, models 1-5 were fitted using quintiles of n-3 PUFA intake from food and supplements at DSA1 as the main exposure (online supplementary appendix III).

The above analyses were repeated using time-updated measures of supplement use and covariates using model 3. First, we performed separate analyses using DSA1, DSA2 and DSA3 as the time origins. Second, we performed time-varying covariates modelling using the full length of follow-up as well as shorter length of follow-up time to acknowledge unobserved changes in individuals' supplement use over time. We equally applied the strategy described here using the change between supplement group categories between DSA.

We assessed interactions between supplement use and sex, age, self-reported illnesses, smoking and BMI using likelihood ratio tests.

At DSA1, participants with missing data on supplement use and covariables were excluded; at DSA2 and DSA3 missing covariates from the last observation were carried forward (figure 1). Alternatively, multiple imputation was used to handle missing data in the Cox regression analyses (supplementary appendix IV). The proportional hazards' assumption was assessed in the time-varying covariates model, by including interactions between time and each explanatory variable. Data were analysed using Stata V.14.

\section{RESULTS}

Analyses were based on 22035 participants who provided data at DSA1, 15937 participants at DSA2 and 10120 participants at DSA3 (figure 1). Median (IQR) of follow-up time was $19(17,20)$ years. During this time, 1562 participants (out of 22 035) died of CHD mentioned anywhere on the death certificate.

\section{Description of included participants}

Table 1 (and online supplementary appendix V) describes the study participants at each DSA. Included participants had a median age of 59, 65 and 69 years at DSA1, DSA2 and DSA3, respectively, and consisted of $45 \%, 43 \%$ and $44 \%$ men. We observed a lower smoking prevalence, fewer alcohol consumers and more physical inactivity as time progressed; however, there was still a large range in these behaviours. The proportion of NSU dropped from $61 \%$ at DSA 1 to $50 \%$ at DSA3, with $24 \%, 31 \%$ and $34 \%$ using n-3 PUFA containing supplements at the three DSA, respectively. The change in supplement use over time is depicted in online supplementary appendix II.

Multinomial logistic regression analysis assessed the relation of these characteristics to supplement use status, mutually adjusted, at DSA1, DSA2 and DSA3 (figure 2). Having a non-manual social class and a higher education level were associated with SU-n3; whereas older participants, non-smoking, alcohol consumption and fewer self-reported illnesses were asso

ciated with $\mathrm{SU}+\mathrm{n} 3$. Associations between covariates and supplement use group were similar at the three DSA.

\section{n-3 PUFA supplement users and hazard of CHD mortality}

There was no evidence that the CHD mortality of SU+n3 or SU-n3 differed from NSU when solely using baseline data (online supplementary appendix VI.). Table 2 shows results from the Cox proportional hazards analyses in which the time origin was reset at each DSA. No association was observed between supplement use at DSA1 and CHD mortality for any amount of follow-up time. On the contrary, the HR of CHD mortality for SU+n3 relative to NSU at DSA2 and DSA3 was significantly below 1 . With increasing follow-up time the HR attenuated, but remained statistically significant. We observed stronger associations, for all lengths of follow-up studied, between $\mathrm{SU}+\mathrm{n} 3$ and CHD mortality in the later DSAs.

Using time-varying analysis, we observed a $26 \%$ lower hazard among SU+n3 compared with NSU (HR: 0.74, $95 \%$ CI 0.66 to 0.84 ). Significant interactions between follow-up time and age, sex and self-reported myocardial infarction were observed; however, inclusion of these interaction terms resulted in negligible differences in the HR or $95 \%$ CI of the main exposure. Results presented are therefore without these interaction terms in the regression equation.

\section{Change in supplement use over time}

Table 3 shows the results from analysis investigating changes in supplement use over time. There was no evidence to suggest that participants who were taking n-3 PUFA supplements at an earlier point in time, but who stopped its use, had a different hazard of CHD mortality compared with consistent NSU ( $\mathrm{HR}_{\mathrm{DSAl}-\mathrm{DSA} 2} 1.24,95 \% \mathrm{CI}$ 0.94 to 1.64 ; $\mathrm{HR}_{\mathrm{DSA} 2-\mathrm{DSA} 3} 1.20,95 \%$ CI 0.79 to 1.81 ), therefore suggesting that there are no long term benefits of previous n-3 PUFA supplement use. Compared with consistent NSU, participants who reported n-3 PUFA 
Table 1 Characteristics of the EPIC-Norfolk cohort at time of DSA1 (1993-1998), DSA2 (2002-2004) and DSA3 (2004-2011)

\begin{tabular}{|c|c|c|c|}
\hline & $\begin{array}{l}\text { DSA1 } \\
n=22035\end{array}$ & $\begin{array}{l}\text { DSA2 } \\
n=15937\end{array}$ & $\begin{array}{l}\text { DSA3 } \\
n=10120\end{array}$ \\
\hline CHD mortality events from DSA onwards ( $n$ ) & 1562 & 742 & 241 \\
\hline Total person years at risk (years) & 383444 & 173239 & 63535 \\
\hline Crude cumulative rate (per 1000 person years) & 4.074 & 4.283 & 3.793 \\
\hline \multicolumn{4}{|l|}{ Supplement use } \\
\hline NSU & $61(13444)$ & 52 (8353) & $50(5029)$ \\
\hline SU-n3 & $15(3263)$ & $17(2665)$ & $16(1610)$ \\
\hline $\mathrm{SU}+\mathrm{n3}$ & $24(5328)$ & $31(4919)$ & $34(3481)$ \\
\hline \multicolumn{4}{|l|}{ n-3 PUFA (g/day)* } \\
\hline From food only & $0.12(0.06-0.34)$ & - & - \\
\hline From food and supplements combined & $0.16(0.07-0.40)$ & - & - \\
\hline Among SU+n3 only & $0.30(0.17-0.72)$ & - & - \\
\hline \multicolumn{4}{|l|}{ Sex } \\
\hline Men & $45(9890)$ & $43(6923)$ & $44(4418)$ \\
\hline Women & $55(12145)$ & 57 (9014) & $56(5702)$ \\
\hline Age (years) & $59(51-67)$ & $65(58-72)$ & $69(63-76)$ \\
\hline BMI $\left(\mathrm{kg} / \mathrm{m}^{2}\right)$ & $25.8(23.7-28.3)$ & $26.1(24.0-28.7)$ & $26.3(24.0-29.0)$ \\
\hline \multicolumn{4}{|l|}{ Smoking status } \\
\hline Current & $11(2395)$ & 7 (1149) & $5(456)$ \\
\hline Former & $43(9426)$ & 50 (7982) & $46(4671)$ \\
\hline Never & 46 (10 214) & $43(6806)$ & 49 (4993) \\
\hline \multicolumn{4}{|l|}{ Social class } \\
\hline Professional & 7 (1531) & 8 (1203) & $8(852)$ \\
\hline Managerial & $37(8048)$ & 39 (6189) & $40(4047)$ \\
\hline Skilled non-manual & $17(3715)$ & $16(2585)$ & $16(1658)$ \\
\hline Skilled manual & $23(5055)$ & $22(3483)$ & $21(2131)$ \\
\hline Semi-skilled & $13(2910)$ & $13(2007)$ & $12(1163)$ \\
\hline Non-skilled & $4(776)$ & $3(470)$ & $3(269)$ \\
\hline \multicolumn{4}{|l|}{ Marital status } \\
\hline Married & $82(18$ 127) & 78 (12 394) & 76 (7708) \\
\hline Not married & $18(3908)$ & $22(3543)$ & $24(2412)$ \\
\hline \multicolumn{4}{|l|}{ Education level } \\
\hline No qualification & 36 (7999) & $32(5106)$ & $28(2851)$ \\
\hline O-level & $10(2282)$ & $11(1757)$ & $12(1184)$ \\
\hline A-level & $41(8955)$ & $42(6720)$ & $44(4427)$ \\
\hline Degree & 13 (2799) & 15 (2354) & $16(1658)$ \\
\hline \multicolumn{4}{|l|}{ Season } \\
\hline Spring & $26(5817)$ & $23(3645)$ & 27 (2761) \\
\hline Summer & 25 (5473) & 21 (3295) & 30 (3008) \\
\hline Autumn & $26(5661)$ & $32(5150)$ & 25 (2513) \\
\hline Winter & $23(5084)$ & $24(3847)$ & $18(1838)$ \\
\hline \multicolumn{4}{|l|}{ Physical activity } \\
\hline Inactive & 30 (6592) & 36 (5809) & $41(4106)$ \\
\hline Moderately inactive & 29 (6389) & $27(4302)$ & $28(2792)$ \\
\hline Moderately active & $23(5040)$ & $20(3151)$ & 17 (1709) \\
\hline
\end{tabular}


Table 1 Continued

\begin{tabular}{|c|c|c|c|}
\hline & $\begin{array}{l}\text { DSA1 } \\
n=22035\end{array}$ & $\begin{array}{l}\text { DSA2 } \\
n=15937\end{array}$ & $\begin{array}{l}\text { DSA3 } \\
n=10120\end{array}$ \\
\hline Active & $18(4014)$ & 17 (2675) & $15(1513)$ \\
\hline \multicolumn{4}{|l|}{ Alcohol intake (HLQ) } \\
\hline None & 13 (2930) & 32 (5042) & 33 (3312) \\
\hline$>0-14$ units/week & $72(15880)$ & 52 (8336) & 56 (5704) \\
\hline$>14-28$ units/week & $11(2418)$ & $12(1950)$ & $9(869)$ \\
\hline$>28$ units/week & $4(807)$ & $4(609)$ & $2(235)$ \\
\hline \multicolumn{4}{|l|}{ Self-reported illness } \\
\hline Myocardial infarction & $3(704)$ & $5(717)$ & $3(330)$ \\
\hline Stroke & $1(304)$ & $2(359)$ & $2(164)$ \\
\hline Diabetes & $2(510)$ & $5(745)$ & $3(338)$ \\
\hline Energy $-7 \mathrm{dDD}(\mathrm{MJ} / \mathrm{day})^{*}$ & $8.00(6.71-9.56)$ & & \\
\hline Protein (en\%) & $15.2(13.6-16.9)$ & & \\
\hline Fat (en\%) & $33.4(29.8-36.8)$ & & \\
\hline Saturated fat (en\%) & $12.7(10.9-14.6)$ & & \\
\hline Carbohydrates (en\%) & $47.6(43.4-51.5)$ & & \\
\hline Alcohol (en\%) & $2.0(0-5.9)$ & & \\
\hline \multicolumn{4}{|l|}{ Food intake-7dDD (g/day) ${ }^{*}$} \\
\hline Fruit & $153(82-242)$ & & \\
\hline Vegetables & $142(102-190)$ & & \\
\hline Red and processed meat & $53(32-76)$ & & \\
\hline White meat & $21(6-37)$ & & \\
\hline Oily fish & $4(0-19)$ & & \\
\hline Consumers only (54\%) & $17(10-30)$ & & \\
\hline White fish & $13(0-23)$ & & \\
\hline Consumers only (66\%) & $19(13-29)$ & & \\
\hline Total fish & $23(11-40)$ & & \\
\hline Consumers only (84\%) & $28(17-44)$ & & \\
\hline
\end{tabular}

Values are \% (n) for categorical variables and median (IQR) for continuous variables.

*Data only available from DSA1, see also online supplementary appendix I; for more information on diet at DSA1, see online supplementary appendix V.

7dDD, 7-day diet diary; BMI, body mass index; CHD, coronary heart disease; DSA, dietary supplement assessment; EPIC, European Prospective Investigation into Cancer; HLQ, Health and Lifestyle Questionnaire; n-3 PUFA, omega-3 polyunsaturated fatty acids (sum of eicosapentaenoic acid and docosahexaenoic acid); NSU, non-supplement user; SU, supplement user.

supplement use at both time points, and participants who became a SU+n3 had a lower hazard of CHD mortality. This association was stronger in the time-varying covariates analysis where we observed a HR of $0.69(95 \% \mathrm{CI}$ 0.52 to 0.90 ) among starters of SU+n3 and a HR of 0.78 (95\% CI 0.63 to 0.98 ) among consistent SU+n3. Associations attenuated or became non-significant as follow-up time increased.

\section{Sensitivity analyses}

Using different definitions for the endpoint (figure 3), we observed associations of similar strength for CHD as underlying cause of death (HR: $0.78,95 \%$ CI 0.67 to 0.90 ) and for acute fatal myocardial infarction when compared with CHD mortality mentioned anywhere on the death certificate. No associations were observed for first event of hospitalisation due to CHD.

Associations between SU+n3and CHD mortality were similar for men and women, for overweight and non-overweight participants, for participants with and without self-reported myocardial infarction or diabetes and among smokers and non-smokers (online supplementary appendix IV). There was evidence of an interaction between supplement use group and prevalent stroke ( $\mathrm{p}=0.006$ ); although the $\mathrm{SU}+\mathrm{n} 3 \mathrm{had}$ similar HRs by prevalent stroke group, SU-n3 had not.

The primary analyses do not distinguish between $\mathrm{SU}+\mathrm{n} 3$ who do or do not use other supplements. As an additional sensitivity analysis, we investigated this using 

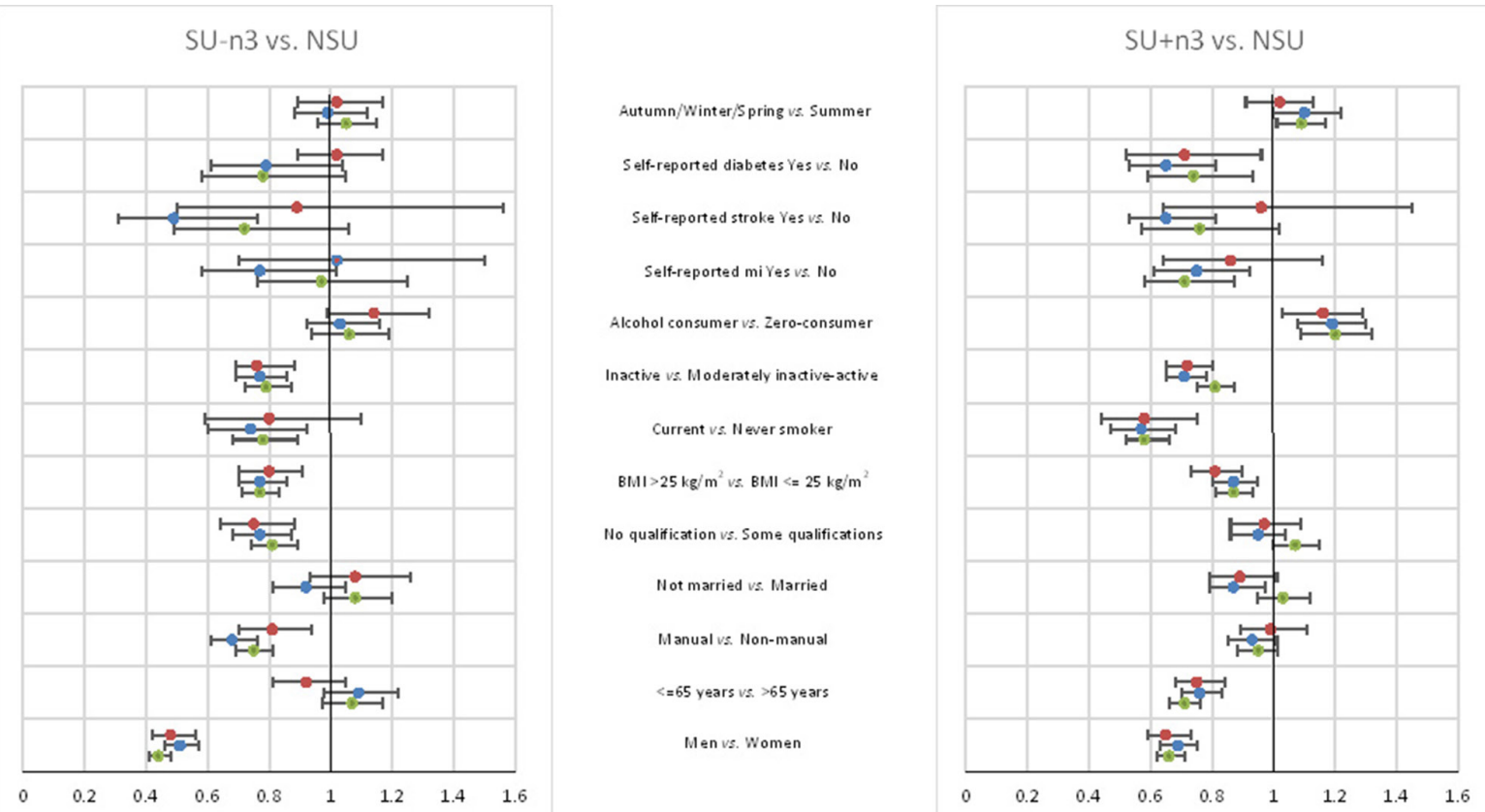

Figure 2 Characteristics of SU-n3 versus NSU and SU+n3 versus NSU measured at three time points (DSA1 [green], DSA2 [blue], DSA3 [red]) in the EPIC-Norfolk study. Three analyses from multinomial logistic regression at three time points: DSA1 $(n=22035)$, DSA2 $(n=12333)$, DSA3 $(n=8126)$. The ORs are mutually adjusted for all variables shown. DSA, dietary supplement assessment; EPIC, European Prospective Investigation into Cancer; NSU, non-supplement user; PUFA, polyunsaturated fatty acids; SU+n3, n-3 PUFA supplement user; SU-n3, supplement user without n-3 PUFA.

a model with binary variables for n-3 PUFA supplement use and non n-3 PUFA supplement use and their interaction, finding that the association between n-3 PUFA supplement use and a reduced hazard persisted in those who used other supplements and those who did not. We found an estimated HR of 0.83 (95\% CI 0.71 to 0.97 ) in those who only use an n-3 PUFA containing supplement versus NSU, a HR of 0.91 (95\% CI 0.78 to 1.06 ) for those who only use non n-3 PUFA containing supplements, and a HR of 0.66 (95\% CI 0.56 to 0.79$)$ versus NSU in those who used both, though there was no evidence that the association between n-3 PUFA containing supplements and CHD was different in those taking no other supplements or in those taking other supplements and the HR in those taking n-3 PUFA supplements adjusted for other supplement use was 0.79 (95\% CI 0.70 to 0.89 ).

\section{DISCUSSION}

\section{Statement of principal findings}

We observed a protective association between n-3 PUFA supplement use and CHD mortality in this general population where fish consumption was low and the mean age was approximately 60 years at baseline (DSA1). When data from three DSA were used, lower hazards of CHD mortality were observed for SU+n3 at all three DSA, which were strongest when the follow-up time was short; however, this analysis lacked statistical power. Using a time-varying covariates analysis, we observed a $26 \%$ lower hazard of CHD mortality among SU+n3 compared with NSU.

\section{Strengths and weaknesses of the study}

A strength is the selection of participants at baseline from a general population as opposed to fish oil trials which have consisted of high-risk populations. ${ }^{11}$ Measures of covariates and supplement use were available from three time points, which enabled mitigation of potential bias due to changes in supplement use over time. We could adjust for several variables representing health behaviours, as well as comorbidities, to account for potential confounding. The DSA had short recall periods of 1 week; however, we have previously shown that measures obtained from three instruments at baseline (DSA1), with recall times varying from 1 year to 1 week, had good agreement. ${ }^{40}$ Supplement use in general is associated with health behaviours ${ }^{41}$ and heterogeneity across supplement users exists, we therefore separated participants into SU+n3 and SU-n3 and carefully assessed potential confounders at all DSA. A further strength was our use of multiple imputation to handle missing data (online supplementary appendix IV.).

The EPIC-Norfolk study has limitations. The baseline response rate was $39 \%$; however, participants were found to be representative of the Health Survey for England population regarding anthropometry, blood pressure and blood lipids. ${ }^{30}$ There was loss to follow-up; however, outcomes were ascertained for those who no longer actively participated. Since the exact dates of supplement use were not known as were the exact dates of self-reported myocardial infarction, stroke or diabetes, we are unable to say which happened first and therefore 


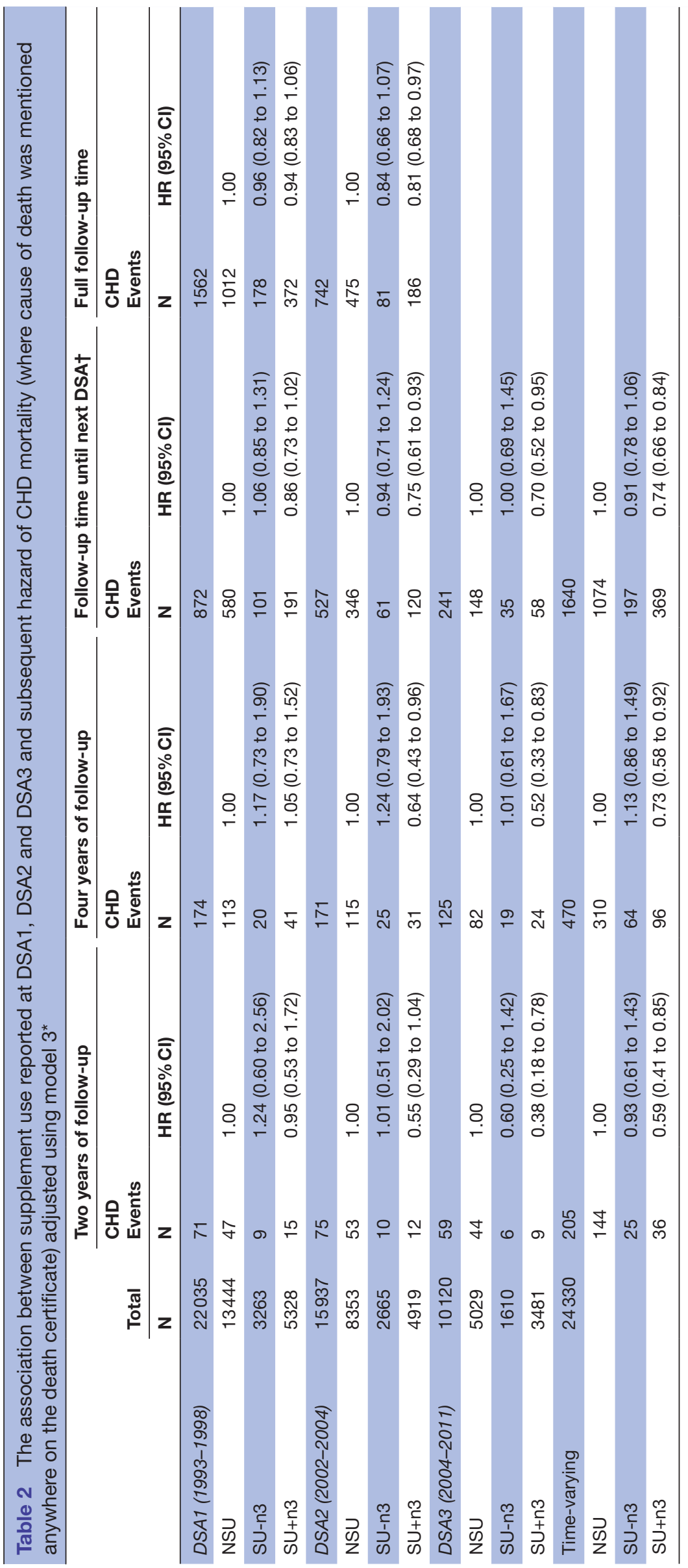

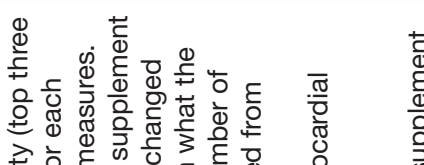

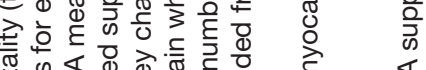

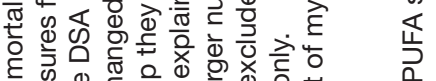

要

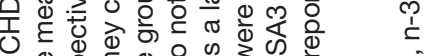

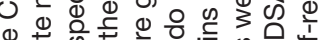

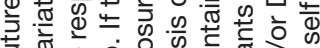

政

응

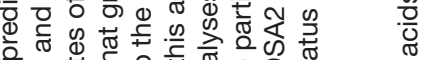

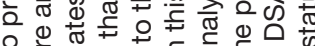

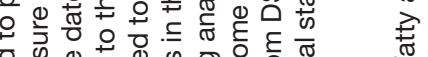

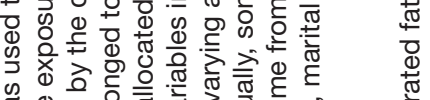

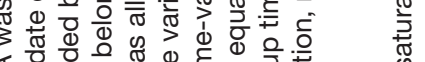

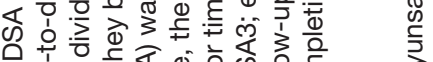

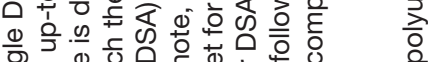

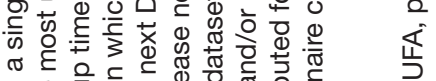

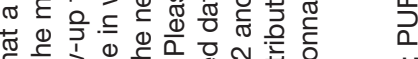

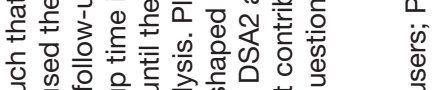

के

का

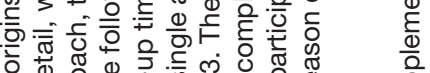

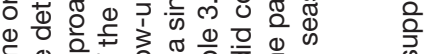

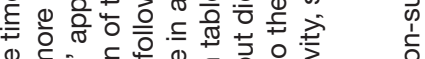

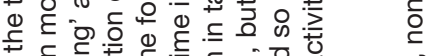

番

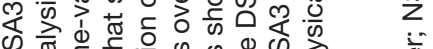

की

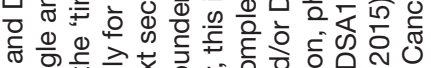

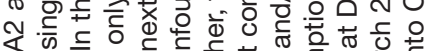

का

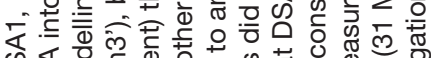

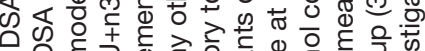

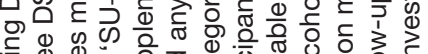

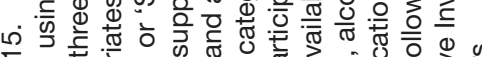

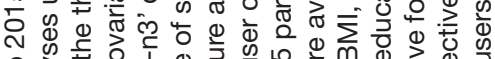

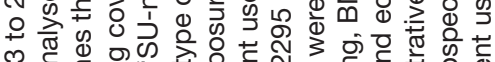

品

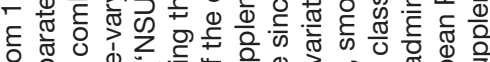

응 응

岁.

分落.

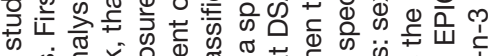

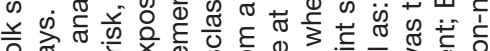

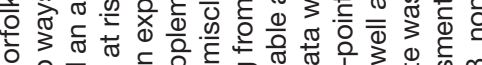

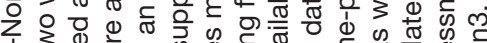

ن

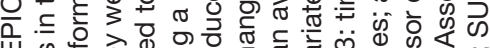

券市

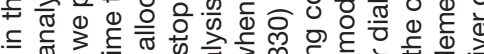

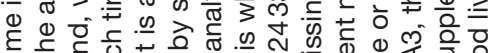

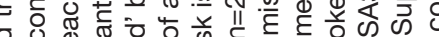

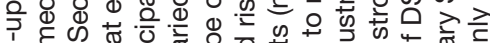

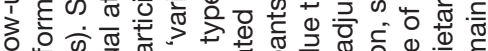

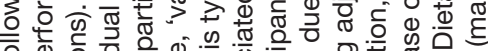

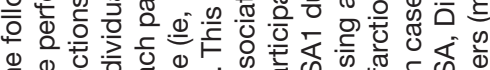

胥

要

$\bar{\omega}$

용

N

$\stackrel{P}{v}$

ڤั)

产

总

혹

콩

항

응

$\frac{D}{\underline{D}}$ 


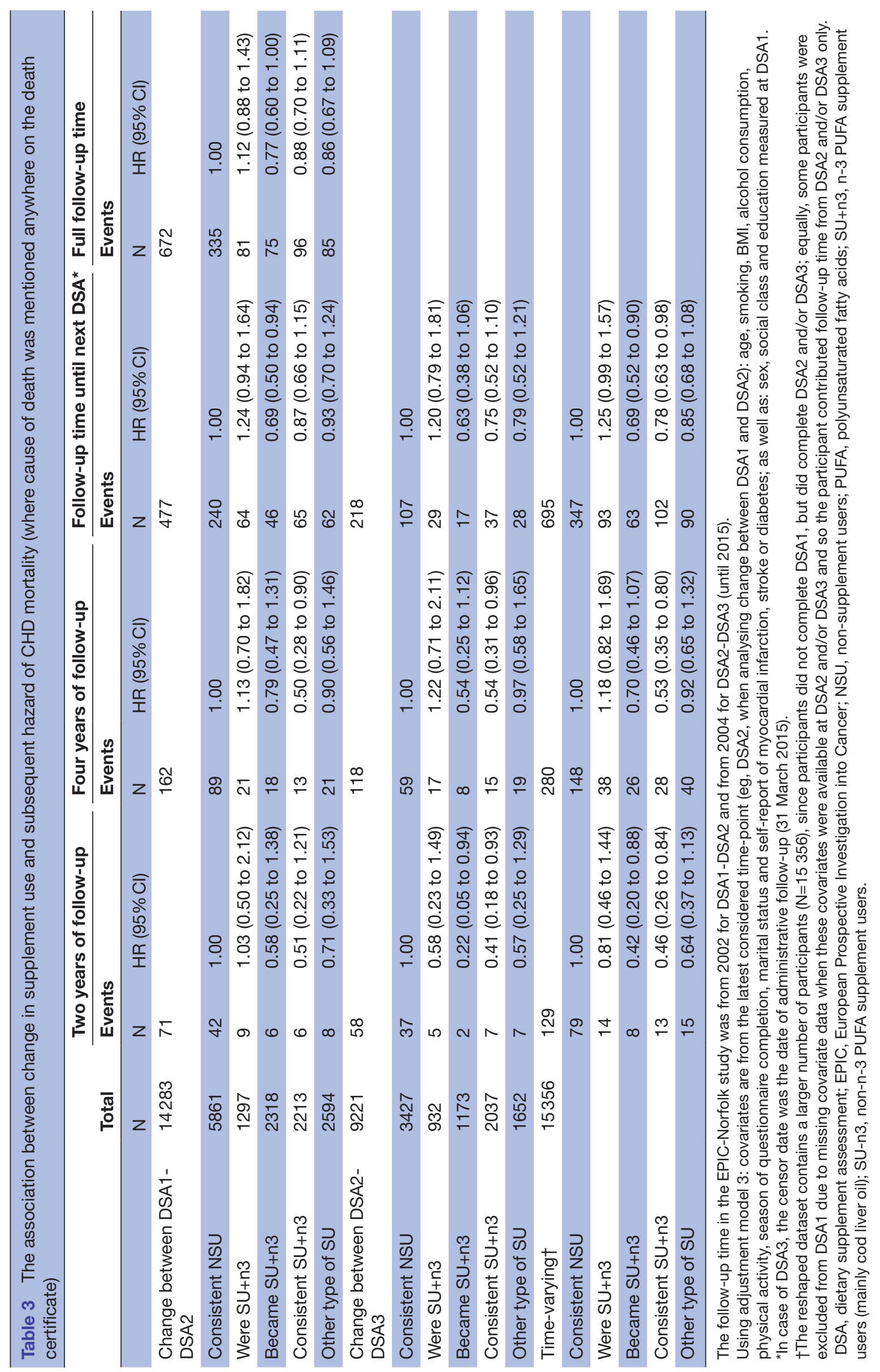




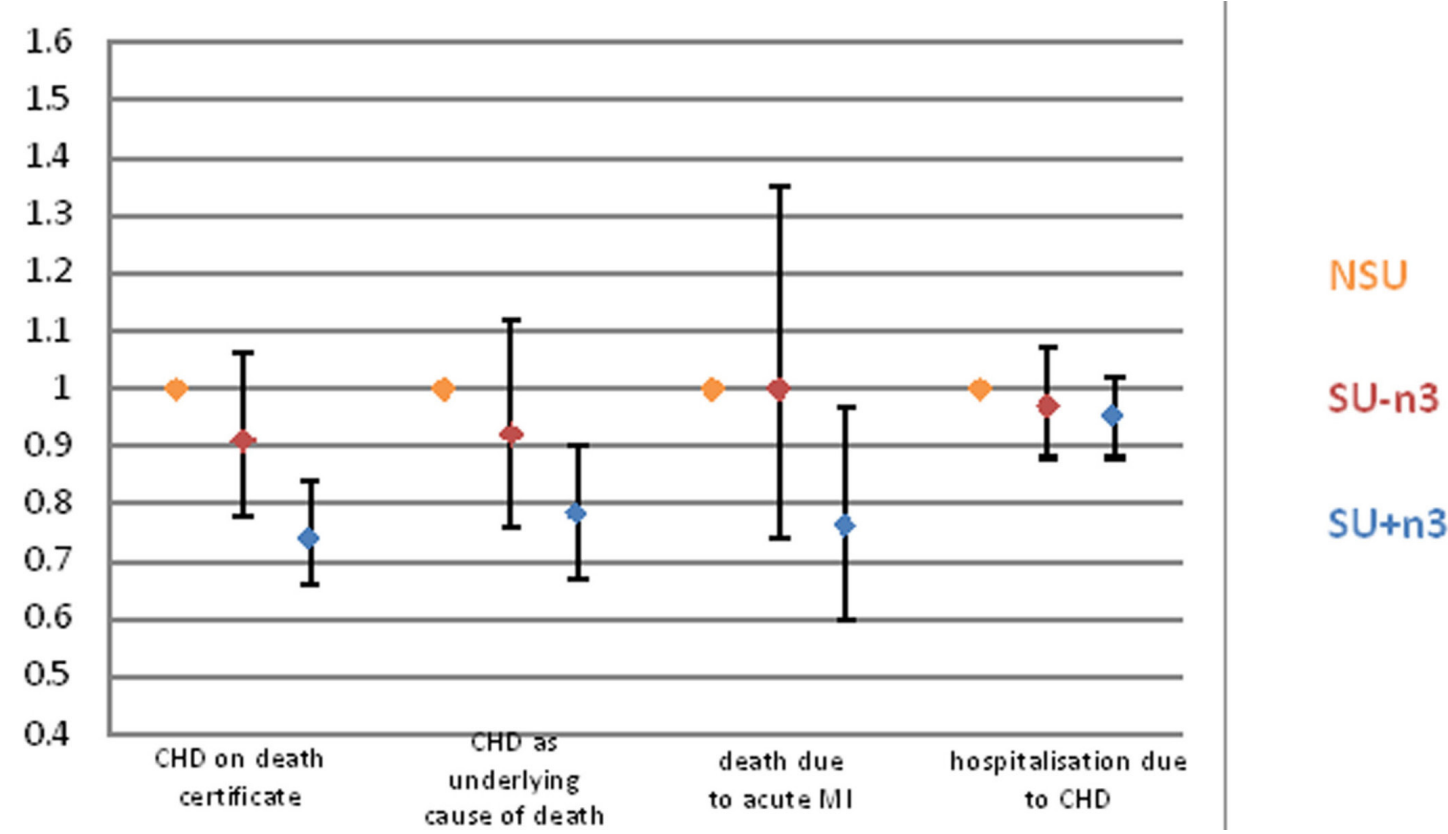

Figure 3 Time-varying covariate analysis of the association between supplement use and hazard of CHD in the EPIC-Norfolk study (follow-up time from 1993 to 2015). (1) CHD mentioned anywhere on the death certificate ( $n=1640 / 24$ 330, as table 2). (2) CHD mentioned as underlying cause of death on death certificate $(n=1084 / 24330)$. (3) Acute myocardial infarction as underlying cause of death on death certificate $(n=411 / 24330)$. (4) Hospitalisation due to CHD ( $n=4087 / 24217)$. The reshaped dataset for mortality analysis contains a larger number of participants $(n=24330)$ than available at DSA1 alone and therefore more events, since participants did not complete DSA1, but did complete DSA2 and/or DSA3; equally, some participants were excluded from DSA1 due to missing covariate data whereas these covariates were available at DSA2 and/or DSA3 and so the participant contributed follow-up time from DSA2 and/or DSA3 only. The reshaped dataset for hospitalisation analysis contains a smaller number of participants $(\mathrm{n}=24216)$ than the mortality analysis, since participants who did not complete DSA1, but completed DSA2 or DSA3 - however had a non-fatal event before DSA2 or DSA3, respectively - were excluded. Using adjustment model 3: time-point specific age, smoking, BMI, alcohol consumption, physical activity, season of questionnaire completion, marital status and self-report of myocardial infarction, stroke or diabetes; as well as: sex, social class and education. NSU, nonsupplement users; SU-n3, non-N-3 PUFA supplement users; SU+n3, N-3 PUFA supplement users (mainly cod liver oil); MI, myocardial infarction.

the chronological ordering is unknown. Healthier individuals might have been more likely to continue active participation; however, we adjusted for a number of factors to mitigate potential bias arising due to this. The time intervals between DSA were relatively long, particularly between DSA1 and DSA2; therefore, misclassification of the exposure is likely to have occurred. Data on fish consumption (and hence n-3 PUFA intake from food) from 7dDD could only be estimated at baseline (online supplementary appendix III). We considered the observed association between the SU+n3 and CHD mortality unlikely to be due to the nutrient/compound content of other non-n-3 PUFA supplements being used, and indeed in sensitivity analyses we found a significant association between n-3 PUFA supplement use and a reduced hazard in those using no other supplements. We also considered it unlikely that the observed associations could be due to the additional vitamins found in n-3 PUFA containing supplements, since these typically contain a $100 \%$ of the recommended nutrient intake and such doses have not been associated with cardiovascular mortality. ${ }^{42}$ Although we found a protective association between SU-n3 and a reduced hazard for CHD mortality in sensitivity analysis $(0.87,95 \%$ CI 0.76 to 0.98$)$, the evidence for this was weak and we were unable to study the wide variety of individual nutrients/compounds in these supplements and we cannot rule out from these results that other supplements could be associated with CHD mortality. Identification of a specific nutrient and biological pathway, as well as the potential for a relative change in the type of supplement consumed among the SU-n3, requires additional analysis with emphasis on confounding by lifestyle and other factors, possibly particularly so if more than one non-n-3 PUFA supplement was consumed. Residual confounding due to dietary differences between SU+n3, SU-n3 and NSU, particularly those occurring over time, such as differences in fruit, vegetables and meat, also cannot be excluded. Lastly, the analyses required interpretation of many models, which will have increased the role of chance findings; however, the significant findings observed may be explained by biological mechanisms, the results were specific to $\mathrm{SU}+\mathrm{n} 3$ and we observed significant associations of starting or continued use of n-3 PUFA supplements with a lower hazard for CHD mortality. 
Meaning of the study: possible explanations and implications for clinicians and policy-makers

The associations observed at the low dose of supplemental EPA and DHA in this cohort makes an antiarrhythmic function of n-3 PUFA, a likely biological pathway. ${ }^{67}$ In time-varying covariate analysis, it was observed that n-3 PUFA supplement use was associated with $26 \%$ lower hazard of CHD mortality compared with non-supplement use. A similar association has been estimated from trial and prospective cohort data ${ }^{6}$ which concluded that $250-500 \mathrm{mg} /$ day of $\mathrm{n}-3$ PUFA lowered risk by $25 \%$ or more; a dose which corresponds to the intake from food and supplements of $300 \mathrm{mg} /$ day observed in this cohort among SU+n3 (table 1). We have previously reported a non-significant association between plasma n-3 PUFA and CHD incidence in a sub-cohort of the EPIC-Norfolk study $^{44}$; however, plasma concentrations were only available at baseline and are likely to have fluctuated over time, partly considering the variability in supplement use (online supplementary appendix II). The results between the studies are consistent when only relying on supplement data from DSA1 in the current analysis (online supplementary appendix VI). The VITAL cohort study found no significant association between increasing quartiles of n-3 PUFA intake from food and supplements and CHD $\left(P_{\text {trend }}=0.120\right)$, although a significant trend was observed for those participants free of baseline CHD $\left(P_{\text {trend }}=0.029\right)$. When including all DSA, effect modification for prevalent myocardial infarction was not observed in EPIC-Norfolk, which might be indicative of the different motivations for CLO use in the UK versus fish oil supplement use in the USA. ${ }^{45}$ In Europe, the highest oily fish consumption is observed in Spain and Scandinavian countries, and is approximately 2-3 fold higher than fish intake in the UK. ${ }^{47}$ A Norwegian cohort, where $30 \%-41 \%$ consumed two or more fish meals per week, observed no association between CLO consumption and CHD mortality. ${ }^{19}$ Fish could therefore have exceeded the threshold for an antiarrhythmic effect in this Norwegian cohort to which CLO could not add, but might supply a considerable contribution to n-3 PUFA intake in the UK. The AGES study in Iceland, where the majority of women consumed 2-4 fish portion per week ( $85 \%$ lean fish), did observe a lower risk of hospitalisation due to CHD when n-3 PUFA from CLO was $\geq 5.9 \mathrm{~g} /$ week, whereas no association was observed with fish consumption. ${ }^{18}$ We did not observe any association with CHD hospitalisation; however, the n-3 PUFA dose in the AGES study was much higher and therefore efficacy might have been through atherosclerotic pathways. Fish oil supplements cannot contain all the nutrients that would be obtained when consuming fish, or indeed the other meal components consumed with it (eg, vegetables) or the red meat replacement that took place, ${ }^{48}{ }^{49}$ but n-3 PUFA supplements contain lower concentrations of contamination, ${ }^{50}$ and provide essential fatty acids without any coating and/or frying oils which might supply trans-fatty acids which are positively associated with CHD. ${ }^{11}$ This is consistent with findings that broiled or baked fish have been associated with a lower risk of CHD mortality, whereas fried fish has not. ${ }^{51}$ Moreover, from DSA1, it is known that n-3 PUFA supplements were consumed on a daily basis by approximately $95 \%$ of the participants. ${ }^{40}$ Fish consumption has not been a daily dietary habit in the UK based on National Survey data in 2000/2001 and 2008/2009-2011/2012. ${ }^{452}$ Incorporation of n-3 PUFA into plasma, platelets and red blood cells has been shown to be enhanced with a daily versus two times a week regimen of similar weekly supplement doses. ${ }^{53}$

\section{Unanswered questions and future research}

Food composition tables which include contaminants (online supplementary appendix III), nutrient composition from supplements and foods and which distinguish between fish preparation methods, could clarify any association between n-3 PUFA sources and CHD to further inform public health dietary guidelines. The results from the first primary prevention trial on n-3 PUFA supplement use are to be expected. ${ }^{29}$

\section{CONCLUSION}

Recent use of n-3 PUFA supplements was associated with a lower hazard of CHD mortality in a population-based cohort with low fish consumption. This association was specific to SU+n3 and independent of history of myocardial infarction.

Acknowledgements The authors thank the EPIC-Norfolk participants and the research and administrative staff for their contributions, past and present.

Contributors MAHL: wrote the manuscript, prepared and analysed both the supplement and dietary data. Developed the research question. RHK: advised on statistical analysis, specifically time-varying covariates analysis and multiple imputation. AAW: Managed the dietary data collection of the study. Contributed to the study protocol. AAM: Prepared dietary data. RNL: Manages study data. Contributed to the study protocol. NJW: Principal investigator of the study. K-TK: Principal investigator of the study. All authors critically read and contributed to the manuscript.

Funding All authors report programme grants from Cancer Research UK (G0401527, G1000143) and the Medical Research Council (MRC) (C864/A8257, C864/A14136) during the study. RHK is supported by an MRC fellowship (MR/ M014827/1). The funders of EPIC-Norfolk had no role in the study design, data collection, data analysis, interpretation of data, writing of the report or in the decision to submit the paper for publication.

Competing interests None declared.

Ethics approval Norwich District Health Authority Ethics Committee.

Provenance and peer review Not commissioned; externally peer reviewed.

Data sharing statement More information on the EPIC-Norfolk study can be obtained from the website: www.epic-norfolk.org.uk. Requests for collaboration will be discussed at the EPIC-Norfolk management meetings.

Open Access This is an Open Access article distributed in accordance with the terms of the Creative Commons Attribution (CC BY 4.0) license, which permits others to distribute, remix, adapt and build upon this work, for commercial use, provided the original work is properly cited. See: http://creativecommons.org/ licenses/by/4.0/ 
(c) Article author(s) (or their employer(s) unless otherwise stated in the text of the article) 2017. All rights reserved. No commercial use is permitted unless otherwise expressly granted.

\section{REFERENCES}

1. Dyerberg J. Fatty acid composition of the plasma lipids in hypothyroid subjects. Acta Med Scand 1968;184:441-9.

2. Kromhout D, Bosschieter EB, de Lezenne Coulander C. The inverse relation between fish consumption and 20-year mortality from coronary heart disease. N Engl J Med 1985;312:1205-9.

3. Zheng J, Huang T, Yu Y, et al. Fish consumption and CHD mortality: an updated meta-analysis of seventeen cohort studies. Public Health Nutr 2012;15:725-37.

4. Henderson L, Gregory J, Swan G. The national diet \& nutrition survey: adults aged 19 to 64 years. Types and quantities of foods consumed. London: Her Majesty's Stationery Office (HMSO), 2002.

5. Lentjes MA, Mulligan AA, Welch AA, et al. Contribution of cod liver oil-related nutrients (vitamins A, D, E and eicosapentaenoic acid and docosahexaenoic acid) to daily nutrient intake and their associations with plasma concentrations in the EPIC-Norfolk cohort. J Hum Nutr Diet 2015;28:568-82.

6. Mozaffarian D, Rimm EB. Fish intake, contaminants, and human health: evaluating the risks and the benefits. JAMA 2006;296:1885-99.

7. London B, Albert C, Anderson ME, et al. Omega-3 Fatty Acids and Cardiac Arrhythmias: Prior Studies and Recommendations for Future Research. Circulation 2007;116:e320-35.

8. Chiuve SE, Rimm EB, Sandhu RK, et al. Dietary fat quality and risk of sudden cardiac death in women. Am J Clin Nutr 2012:96:498-507.

9. Hooper L, Thompson RL, Harrison RA, et al. Risks and benefits of omega 3 fats for mortality, cardiovascular disease, and cancer systematic review. BMJ 2006;332:752-60.

10. Rizos EC, Ntzani EE, Bika E, et al. Association between omega-3 fatty acid supplementation and risk of major cardiovascular disease events: a systematic review and meta-analysis. JAMA 2012;308:1024-33.

11. Chowdhury R, Warnakula S, Kunutsor S, et al. Association of dietary, circulating, and supplement fatty acids with coronary risk: a systematic review and meta-analysis. Ann Intern Med 2014;160:398-406.

12. Mozaffarian D, Wu JH, Jhy W. Omega-3 fatty acids and cardiovascular disease: effects on risk factors, molecular pathways, and clinical events. J Am Coll Cardiol 2011;58:2047-67.

13. Wu JH, Mozaffarian D. $\omega-3$ fatty acids, atherosclerosis progression and cardiovascular outcomes in recent trials: new pieces in a complex puzzle. Heart 2014;100:530-3.

14. James MJ, Sullivan TR, Metcalf RG, et al. Pitfalls in the use of randomised controlled trials for fish oil studies with cardiac patients. Br J Nutr 2014;112:812-20.

15. Ridker PM. Fish consumption, fish oils, and cardiovascular events: still waiting for definitive evidence. Am J Clin Nutr 2016;104:951-2.

16. Skeie G, Braaten T, Hjartåker A, et al. Use of dietary supplements in the European Prospective Investigation into Cancer and Nutrition calibration study. Eur J Clin Nutr 2009;63(Suppl 4):S226-S238.

17. Messerer M, Håkansson N, Wolk $A$, et al. Dietary supplement use and mortality in a cohort of Swedish men. Br J Nutr 2008;99:626-31.

18. Haraldsdottir A, Torfadottir JE, Valdimarsdottir UA, et al. Fish and fish-liver oil consumption in adolescence and midlife and risk of $\mathrm{CHD}$ in older women. Public Health Nutr 2016;19:318-25.

19. Egeland GM, Meyer HE, Selmer R, et al. Cod liver oil consumption, smoking, and coronary heart disease mortality: three counties, Norway. Int J Circumpolar Health 2001;60:143-9.

20. Mozaffarian D, Lemaitre RN, King IB, et al. Plasma phospholipid long-chain $\omega-3$ fatty acids and total and cause-specific mortality in older adults: a cohort study. Ann Intern Med 2013;158:515-25.

21. Bell GA, Kantor ED, Lampe JW, et al. Intake of long-chain $\omega-3$ fatty acids from diet and supplements in relation to mortality. Am J Epidemiol 2014:179:710-20.

22. Yokoyama M, Origasa $\mathrm{H}$, Matsuzaki M, et al. Effects of eicosapentaenoic acid on major coronary events in hypercholesterolaemic patients (JELIS): a randomised open-label, blinded endpoint analysis. Lancet 2007;369:1090-8.

23. The Origin Trial Investigators. Basal Insulin and Cardiovascular and Other Outcomes in Dysglycemia. N Engl J Med 2012;367:319-28.

24. Roncaglioni MC, Tombesi M, Avanzini F, et al. n-3 fatty acids in patients with multiple cardiovascular risk factors. $N$ Engl J Med 2013;368:1800-8.
25. Burr ML. Secondary prevention of CHD in UK men: the Diet and Reinfarction Trial and its sequel. Proc Nutr Soc 2007;66:9-15.

26. Investigators G-P. Dietary supplementation with $n-3$ polyunsaturated fatty acids and vitamin $E$ after myocardial infarction: results of the GISSI-Prevenzione trial. Gruppo Italiano per lo Studio della Sopravvivenza nell'Infarto miocardico. Lancet 1999;354:447-55.

27. Rauch B, Schiele R, Schneider S, et al. OMEGA, a randomized, placebo-controlled trial to test the effect of highly purified omega-3 fatty acids on top of modern guideline-adjusted therapy after myocardial infarction. Circulation 2010;122:2152-9.

28. Galan P, Kesse-Guyot E, Czernichow S, et al. Effects of B vitamins and omega 3 fatty acids on cardiovascular diseases: a randomised placebo controlled trial. BMJ 2010;341:c6273.

29. Manson JE, Bassuk SS, Lee IM, et al. The VITamin D and OmegA-3 TriaL (VITAL): rationale and design of a large randomized controlled trial of vitamin D and marine omega-3 fatty acid supplements for the primary prevention of cancer and cardiovascular disease. Contemp Clin Trials 2012;33:159-71.

30. Day N, Oakes S, Luben R, et al. EPIC-Norfolk: study design and characteristics of the cohort. European Prospective Investigation of Cancer. Br J Cancer 1999;80(Suppl 1):95-103.

31. Hayat SA, Luben R, Keevil VL, et al. Cohort profile: A prospective cohort study of objective physical and cognitive capability and visual health in an ageing population of men and women in Norfolk (EPICNorfolk 3). Int J Epidemiol 2014;43:1063-72.

32. European Commission. Directive 2002/46/EC of the European Parliament and of the Council of 10 June 2002 on the approximation of the laws of the Member States relating to food supplements. Off $J$ Eur Communities 2002;L183/51.

33. Lentjes MA, Bhaniani A, Mulligan AA, et al. Developing a database of vitamin and mineral supplements (ViMiS) for the Norfolk arm of the European Prospective Investigation into Cancer (EPIC-Norfolk). Public Health Nutr 2011;14:459-71.

34. Bingham SA, Welch AA, McTaggart A, et al. Nutritional methods in the European Prospective Investigation of Cancer in Norfolk. Public Health Nutr 2001;4:847-58.

35. Welch AA, McTaggart A, Mulligan AA, et al. DINER (Data Into Nutrients for Epidemiological Research) - a new data-entry program for nutritional analysis in the EPIC-Norfolk cohort and the 7-day diary method. Public Health Nutr 2001;4:1253-65.

36. Lentjes MA, McTaggart A, Mulligan AA, et al. Dietary intake measurement using $7 \mathrm{~d}$ diet diaries in British men and women in the European Prospective Investigation into Cancer-Norfolk study: a focus on methodological issues. Br J Nutr 2014;111:516-26.

37. Holland B, Welch AA, Unwin D, et al. McCance and Widdowson's the composition of foods. Cambridge: Royal Society of Chemistry (RSC), 1991.

38. Mulligan AA, Kuhnle GG, Lentjes MA, et al. Intakes and sources of isoflavones, lignans, enterolignans, coumestrol and soyacontaining foods in the Norfolk arm of the European Prospective Investigation into Cancer and Nutrition (EPIC-Norfolk), from $7 \mathrm{~d}$ food diaries, using a newly updated database. Public Health Nutr 2013;16:1454-62.

39. Welch AA, Shakya-Shrestha S, Lentjes MA, et al. Dietary intake and status of $n-3$ polyunsaturated fatty acids in a population of fisheating and non-fish-eating meat-eaters, vegetarians, and vegans and the precursor-product ratio of -linolenic acid to long-chain n-3 polyunsaturated fatty acids: results from the EPIC-Norfolk cohort. Am J Clin Nutr 2010;92:1040-51.

40. Lentjes MA, Welch AA, Luben RN, et al. Differences in dietary supplement use and secular and seasonal trends assessed using three different instruments in the EPIC-Norfolk population study. $J$ Diet Supp/ 2013;10:142-51.

41. Lentjes MA, Welch AA, Mulligan AA, et al. Cod liver oil supplement consumption and health: cross-sectional results from the EPICNorfolk cohort study. Nutrients 2014;6:4320-37.

42. Macpherson H, Pipingas A, Pase MP. Multivitamin-multimineral supplementation and mortality: a meta-analysis of randomized controlled trials. Am J Clin Nutr 2013;97:437-44.

43. Myung SK, Ju W, Cho B, et al. Efficacy of vitamin and antioxidant supplements in prevention of cardiovascular disease: systematic review and meta-analysis of randomised controlled trials. BMJ 2013;346:f10.

44. Khaw KT, Friesen MD, Riboli E, et al. Plasma phospholipid fatty acid concentration and incident coronary heart disease in men and women: the EPIC-Norfolk prospective study. PLoS Med 2012;9:e1001255.

45. Kim HJ, Giovannucci E, Rosner B, et al. Longitudinal and secular trends in dietary supplement use: Nurses' Health Study and Health Professionals Follow-Up Study, 1986-2006. J Acad Nutr Diet 2014:114:436-43. 
46. Rajakumar K. Vitamin D, cod-liver oil, sunlight, and rickets: a historical perspective. Pediatrics 2003;112:e132-e135.

47. Welch AA, Lund E, Amiano P, et al. Variability of fish consumption within the 10 European countries participating in the European Investigation into Cancer and Nutrition (EPIC) study. Public Health Nutr 2002;5:1273-85.

48. Kiefte-de Jong JC, Chowdhury R, Franco OH. Fish intake or omega-3 fatty acids: greater than the sum of all parts? Eur $J$ Epidemiol 2012;27:891-4.

49. He K, Fish HK. Fish, long-chain omega-3 polyunsaturated fatty acids and prevention of cardiovascular disease--eat fish or take fish oil supplement? Prog Cardiovasc Dis 2009;52:95-114.

50. Bourdon JA, Bazinet TM, Arnason TT, et al. Polychlorinated biphenyls (PCBs) contamination and aryl hydrocarbon receptor (AhR) agonist activity of Omega-3 polyunsaturated fatty acid supplements: implications for daily intake of dioxins and PCBs. Food Chem Toxicol 2010;48:3093-7.

51. Mozaffarian D, Lemaitre RN, Kuller LH, et al. Cardiac benefits of fish consumption may depend on the type of fish meal consumed: the Cardiovascular Health Study. Circulation 2003; 107:1372-7.

52. Bates B, Lennox A, Prentice A, eds. National Diet and Nutrition Survey Results from Years 1, 2, 3 and 4 (combined) of the Rolling Programme (2008/2009-2011/2012). London: Public Health England, 2014.

53. Browning LM, Walker CG, Mander AP, et al. Compared with daily, weekly n-3 PUFA intake affects the incorporation of eicosapentaenoic acid and docosahexaenoic acid into platelets and mononuclear cells in humans. J Nutr 2014;144:667-72. 